\title{
Potenciales mecanismos de neuroinvasión del SARS-CoV-2: una revisión de la literatura actual.
}

Potential neuroinvasion pathways of SARS-CoV-2: a review of the current literature.

Rafael Alarco ${ }^{1, \text { a }}$, Jeff Huarcaya-Victoria ${ }^{2,3, b}$

\section{RESUMEN}

Desde su inicio, en diciembre del 2019, la pandemia causada por el nuevo coronavirus 2019 (COVID-19), ha infectado a más de 116 millones de personas. A las bien documentadas manifestaciones respiratorias causadas por el SARS-CoV-2, se está añadiendo un creciente número de manifestaciones neurológicas y psiquiátricas entre los pacientes afectados y sobrevivientes. En este artículo se revisan y describen los potenciales mecanismos de invasión del virus al sistema nervioso. Sobre la base de estudios precedentes en coronavirus similares (MERS-CoV y SARS-CoV) y la evidencia actual, se plantea que las posibles rutas de neuroinvasión que emplea el SARS-CoV-2 son la transneuronal (vía axonal retrógrada, a través de los nervios periféricos), la hematógena/linfática (libre a través de la sangre y linfa o en el interior de las células inmunes) y la digestiva (mediante disrupción de la barrera intestinal). Si bien es necesario conducir más investigaciones en varias áreas, dilucidar las rutas de neuroinvasión, así como las capacidades neurotrópicas del virus, son puntos de vital importancia para entender y tratar las múltiples manifestaciones neurológicas y psiquiátricas, así como las potenciales secuelas a largo plazo que los pacientes infectados puedan desarrollar.

PALABRAS CLAVE: SARS-CoV-2; coronavirus; COVID-19; neuropsiquiatría; fisiopatología; sistema nervioso

\section{SUMMARY}

Since its beginning, in December 2019, the pandemic caused by the coronavirus disease 2019 (COVID-19) has infected more than 116 millions of people. In addition to the well-documented respiratory manifestations caused by SARS-CoV-2, an increasing number of neurological and psychiatric manifestations are being reported among affected individuals and survivors. In this article we describe the potential invasion mechanisms of SARS-CoV-2 to the nervous system. On the basis of preceding studies on similar coronaviruses (MERS-CoV and SARS-CoV) and current evidence, three possible neuroinvasion routes used by SARS-CoV-2 are suggested: the transneuronal pathway (retrograde axonal pathway through the peripheral nerves), the hematogenous / lymphatic pathway (free through the blood and lymph or inside immune cells), and the digestive pathway (through disruption of the intestinal barrier). Although more research is needed in several areas, the elucidation of neuroinvasion routes and

Facultad de Medicina Humana, Universidad de Piura. Lima, Perú.

2 Centro de Investigación en Salud Pública, Facultad de Medicina, Universidad de San Martín de Porres. Lima, Perú.

3 Departamento de Psiquiatría, Hospital Nacional Guillermo Almenara Irigoyen, EsSalud. Lima, Perú.

a Estudiante, https://orcid.org/0000-0002-6654-6036

b Médico psiquiatra, https://orcid.org/0000-0003-4525-9545 
of the neurotropic capacities of the virus, are vital to understand and treat the multiple neurological and psychiatric manifestations as well as the long-term sequelae that the infected patients may potentially develop.

KEYWORDS: SARS-CoV-2; coronavirus; COVID-19; neuropsychiatry; pathophysiology; nervous system

\section{INTRODUCCIÓN}

Desde su identificación en diciembre del 2019 en la ciudad de Wuhan de la República Popular China hasta el 09 de marzo del 2021, la pandemia por la enfermedad causada por coronavirus 2019 (COVID-19) ha cobrado más de 2,5 millones de vidas e infectado a más de 116 millones de personas a nivel mundial (1). Conforme se ha ido conociendo más acerca de esta enfermedad, se han constatado las múltiples manifestaciones de esta, así como la amplia gama de sistemas afectados. Las manifestaciones respiratorias se encuentran bien documentadas. Adicionalmente, un creciente número de estudios están reportando las complicaciones de la COVID-19 a nivel del sistema nervioso central (SNC) (2). Esto nos da indicios de la capacidad neurotrópica del SARS-CoV-2 así como las posibles secuelas neurológicas y psiquiátricas que puede dejar a largo plazo (3).

Como antecedentes directos tenemos las epidemias de los coronavirus MERS-CoV y SARS-CoV. A pesar de no haber muchas investigaciones al respecto (4), se encontraron hallazgos anormales en el tronco encefálico y tálamo en las autopsias de pacientes infectados con MERS-CoV (5), de igual forma un estudio retrospectivo indicó que el $17,4 \%$ de los infectados presentaban síntomas neurológicos (6). Por otra parte, en cerebros post mortem de infectados con SARS-CoV encontraron partículas virales y alteraciones histopatológicas tanto en neuronas como en células gliales $(7,8)$, además de reportarse alteraciones neurológicas (9) y psiquiátricas (10), a largo plazo entre los sobrevivientes. Teniendo en cuenta las similitudes genómicas y fisiopatológicas del SARS-CoV-2 con el SARS-CoV, se espera que las rutas neuroinvasivas sean similares (11).

El SARS-CoV-2, al igual que el resto de coronavirus, es un virus de ARN monocatenario positivo con envoltura (12). Su mecanismo de invasión a la célula huésped dependerá del cebado de la glicoproteína espiga (S) por la proteína de serina transmembrana tipo II (TMPRSS2), y su unión al receptor de la enzima convertidora de angiotensina II (ECAII) (13). Siendo así posibles blancos terapéuticos (14-16). Una vez invadida la célula hospedera puede llegar a causar un cuadro severo caracterizado por insuficiencia respiratoria, sepsis, falla multiorgánica e inflamación sistémica.

Se ha planeado que la expresión de la ECAII por parte del sistema nervioso, sería una posible ruta de entrada del SARS-CoV-2 (4, 17-18). A pesar que la mayoría de signos y síntomas neurológicos son considerados leves (19), han sido reportadas complicaciones mayores como encefalitis o síndrome de Guillan-Barre por inmunocomplejos posiblemente. Asimismo, desde el ámbito psiquiátrico han sido reportados desde trastornos depresivos y ansiosos, hasta episodios psicóticos, delirium y trastorno de estrés postraumático (TEPT) en pacientes infectados (20). Dichas manifestaciones, serán más prevalentes y graves en pacientes con infección severa. Además, se reconoce la importancia de los antecedentes personales, interacciones psicosociales, inflamatorias, inmunológicas, farmacológicas como intervinientes en la infección de SARS-CoV-2 (21).

En la presente revisión abordaremos las principales rutas propuestas de invasión por parte del SARSCoV-2 al sistema nervioso, específicamente al sistema nervioso central.

\section{Búsqueda y selección de artículos}

Para la elaboración de esta revisión se siguieron los lineamientos de la guía PRISMA Statement (22). Se realizó una revisión narrativa, a partir de una búsqueda en PubMed de todos los artículos desde el 11 de agosto del 2003 hasta el 28 de agosto de 2020. Se emplearon los términos: (brain OR neuroinvasive OR neurotropic OR nervous system) AND (SARSCoV-2 OR COVID-19 OR MERS-CoV OR SARSCoV OR coronavirus). Se incluyeron los artículos que contenían información relacionada a los mecanismos de neuroinvasión del SARS-CoV-2, MERS-CoV y SARS-CoV escritos en idioma inglés o español. Se revisaron los títulos y resúmenes de los artículos seleccionados.

De los 1871 artículos encontrados se descartaron 1823 por no cumplir con los criterios de selección. Luego se excluyeron 4 (23-26) artículos debido a falta 


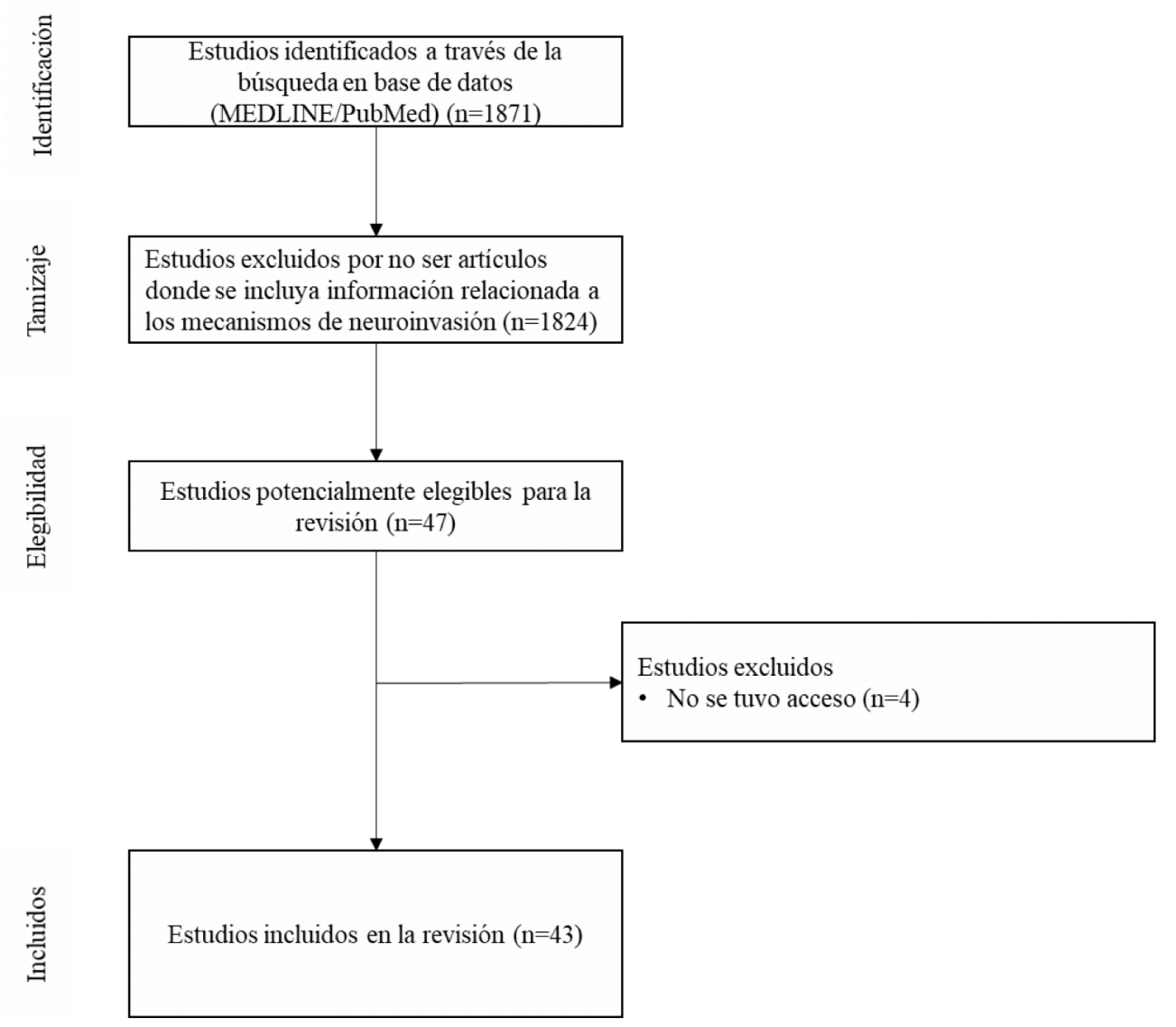

Figura 1. Diagrama de flujo del proceso de identificación y selección de artículos

de acceso. Finalmente, se incluyeron 43 artículos (221, 27-49) (figura 1).

\section{Mecanismos propuestos para la infección del SARS-CoV-2 al SNC}

Dada la evidencia disponible, los principales postulados sobre el mecanismo de infección del SARS-CoV-2 en el cerebro son los siguientes:

\section{Ruta transneuronal}

La evidencia actual sugiere que el SARS$\mathrm{CoV}-2$, invade los nervios periféricos y se disemina vía retrógrada a través de los axones y espacios sinápticos entre las neuronas, de esa manera invade el SNC, y por ende, al cerebro (4). Se ha constatado que diversos coronavirus así como el herpes simplex utilizan el transporte axonal retrógrado para infectar el soma neuronal (27), ello representa una ventaja dado que una vez dentro de las neuronas escapan significativamente de la "vigilancia inmunológica", que será afectada sobretodo en pacientes con leucopenia severa o inmunosuprimidos (11), esto podría explicar por qué los pacientes que presentan los síntomas neuropsiquiátricos más graves son aquellos críticamente enfermos (28). Además en autopsias se han encontrado partículas virales dentro de vesículas dilatadas en neuronas del lóbulo frontal de pacientes con la COVID-19 (28).

Durante el avance de la COVID-19, diversos estudios han reportado trastornos del gusto y olfato como signos de infección inicial por SARS-CoV-2 (29), así como los principales síntomas neurológicos (19). Un estudio llevado a cabo en Italia reportó que la disfunción quimio-sensorial estaba presente en el $19.4 \%$ de los pacientes con COVID-19 (31), mientras un estudio multicéntrico realizado en 12 hospitales europeos determinó que la prevalencia de disfunciones gustativas y olfativas en pacientes con síntomas de moderado-severos, fue del $85.6 \%$ y $88 \%$ respectivamente (19). 
La explicación más plausible a este fenómeno, es la entrada del virus al SNC vía axonal retrógrada del nervio olfatorio. Modelos animales han demostrado grandes concentraciones de ECAII y TMPRSS2 en células no neuronales del epitelio olfatorio, específicamente en las células sustentaculares, pero en cantidades reducidas en las neuronas receptoras olfativas (32). También se ha demostrado un aumento de la expresión de ECAII y TMPRSS2 con la edad, siendo una explicación al porque los pacientes de mayor edad son más susceptibles a infectarse de SARS-CoV-2. Estos hallazgos sugieren que el epitelio olfatorio será una puerta de entrada del SARS-CoV-2, y fruto de la necrosis e inflamación del tejido se ocasionarán los trastornos gustativos y olfativos como la ageusia y anosmia. Posteriormente dependiendo de la vulnerabilidad individual del paciente, infectará al nervio olfatorio, y por vía retrógrada llegará al bulbo olfatorio para de ahí diseminarse al SNC $(4,33)$. Este mecanismo de infección se ve respaldado por un estudio prospectivo con 69 pacientes recuperados de la COVID-19, en los cuales se evidenció una hipertrofia en la corteza olfatoria primaria fruto de una neurogénesis posterior a la infección viral (demostrando que hubo muerte neuronal por la infección del SARS-CoV-2) (2).

Además, se ha reportado infección al bulbo raquídeo por el SARS-CoV-2. Se sostiene que el virus ingresaría por medio de los quimiorreceptores y mecanoreceptores presentes en los pulmones o el nervio neumogástrico (véase ruta digestiva). Mediante vía retrógrada infectará al centro cardiorrespiratorio acrecentando la falla respiratoria, y, por ende, la mortalidad de los pacientes con COVID-19 (11, 34). Sin embargo, esta hipótesis aún es discutida dado que el cuadro no coincide con las características propias de una insuficiencia respiratoria de origen neurológico (35).

Igualmente, un estudio de casos reportó el desarrollo de neuropatía trigeminal de una paciente coinfectada con el virus del herpes zóster y SARSCoV-2, planteando la posibilidad de la infección del nervio trigémino fruto de un sinergismo como posible ruta del SARS-CoV-2 para diseminarse al SNC. Sin embargo, se necesitan hacer más estudios para poder determinar la relación causal (36).

\section{Ruta hematógena/linfática}

Durante la epidemia del SARS-CoV, se determinó que tanto los leucocitos (granulocitos, linfocitos y monocitos) como las células endoteliales expresaban el receptor ECAII (37). Autopsias hechas a pacientes afectados por la COVID-19 han demostrado la presencia de partículas virales tanto en las células endoteliales como en las neuronas del lóbulo frontal adyacentes a esta (29). De igual forma la leucopenia, tormenta de citoquinas y coagulopatías, que pueden desarrollar los pacientes críticamente enfermos, apoyan que la hipótesis de que el SARS-CoV-2 puede infectar a los leucocitos (38).

Se han postulados dos posibles mecanismos por los cuales el SARS-CoV-2 puede atravesar la barrera hemato-encefálica (BHE) y así infectar al SNC:

1) Dada la capacidad del virus de dañar el tejido endotelial del sitio de infección primario, ya sean los alvéolos o el intestino, además de incrementar la permeabilidad vascular junto con la desregulación del sistema renina-angiotensina-aldosterona (SRAA) e inflamación, podrá acceder tanto al torrente sanguíneo como linfático (a través de los ganglios pulmonares y traqueales como se encontró en autopsias de pacientes con SARS-CoV) (39), y de ahí diseminarse a otros órganos expresores de ECAII como el corazón, riñón, hígado o cerebro (33). Una vez que el virus llegue a la BHE, infectará a las células endoteliales que la componen para migrar por mecanismos de endocitosis o exocitosis a las neuronas y glías adyacentes para comenzar su ciclo de replicación degenerando en daño e inflamación dentro del SNC, de una manera similar al que realizan los Arbovirus para cruzar la BHE e ingresar al SNC $(4,40,41)$.

2) El segundo mecanismo es pasar la BHE por medio de leucocitos, células dendríticas y macrófagos infectados, este mecanismo es similar al que el virus de la inmunodeficiencia humana (VIH) utiliza para ingresar al SNC y causar complicaciones neurológicas como la encefalitis (42). Se le conoce como el "mecanismo Caballo de Troya", dado que "burlará" a la selectividad de la BHE al pasar dentro de una célula del sistema inmune de forma similar al que herpes simplex hace con las neuronas (4). Otra ventaja al igual que el SARS-CoV, es que le permitirá al virus mediar en la comunicación inmunitaria, disminuyendo la expresión de citoquinas como el IFN- $\alpha / \beta$ pero aumentando la expresión de citoquinas pro infamatorias como la IL-6 o TNF acrecentando la tormenta de citoquinas (43). Además, este mecanismo se encuentra respaldado por el aumento 
de la permeabilidad en la BHE y con ello una reducción de su selectividad debido a la inflamación sistémica y edema (33).

\section{Ruta digestiva}

El sistema digestivo, al igual que los pulmones y cerebro, expresa el receptor ECAII (11), por ende, es un blanco de infección y replicación del SARS$\mathrm{CoV}-2$. En un estudio se han aislado partículas virales en heces de pacientes con COVID-19 defendiendo la posible ruta de transmisión fecal-oral (44). Un estudio retrospectivo realizado en 651 pacientes chinos con COVID-19 determinó que el 11,4\% presentaron síntomas gastrointestinales (45), mientras un estudio observacional realizado en Perú, determinó que el $4,35 \%$ de los pacientes presentaron vómito y diarrea (46). Los síntomas más reportados han sido vómitos y diarrea, mientras el órgano digestivo más afectado ha sido el intestino. Sin embargo, en pacientes severos también se han reportado patologías como la gastroenteritis viral, hemorragias digestivas bajas y disfunción hepática $(47,48)$.

Al igual que los enterovirus, que iniciarán su proceso patogénico mediante la infección y replicación dentro de las células del epitelio intestinal, se hipotetiza que el SARS-CoV-2 interactuará con los receptores ECAII del epitelio e ingresará al organismo. La infección y replicación viral en el intestino, ocasionará necrosis celular, inflamación y disbiosis que resultarán en una disrupción de la barrera intestinal, que se ha visto comprobado con el aumento de la caliprotectina fecal y disminución de la expresión de ZO-1, ocludina y claudina 3, y la presencia de ARN de SARS-CoV-2 a nivel fecal $(11,49)$. Con a la barrera intestinal comprometida, el virus pasará directamente a la circulación sanguínea o linfática llegando al cerebro como se ha explicado en la ruta hematógena/ linfática anteriormente. Además, se ha propuesto que el virus puede infectar de forma directa las terminaciones nerviosas y llegar vía retrógrada axonal al nervio neumogástrico, por medio del cual podrá comprometer estructuras como el núcleo del tracto solitario o tronco encefálico, lo cual es respaldado por un reciente estudio que muestra gran cantidad de expresión de receptor ECAII en esas regiones (17). Como se mencionó anteriormente se ha propuesto que una invasión en dichas regiones del SNC pueden exacerbar la insuficiencia respiratoria, vómito y alterar la frecuencia cardiaca $(11,34)$. Aún no hay evidencia directa de la capacidad del SARS-CoV-2 para invadir el SNC por el nervio neumogástrico, y la exacerbación de la sintomatología del paciente fruto de la infección al SNC por el SARS-CoV-2 es discutida (35).

\section{Limitaciones}

Debido a que hasta el momento la demostración de la presencia del SARS-CoV-2 en los tejidos nerviosos es escasa, las manifestaciones neuropsiquiátricas de los casos agudos reportados atribuidos al SARSCoV-2 podrían ser una asociación espúrea. Además, la falla para detectar al SARS-CoV-2 en el líquido cefaloraquídeo en pacientes diagnosticados con encefalitis relacionada a la COVID-19, a pesar de la evidente inflamación cerebral, sugieren mecanismos inmuno-mediados en ausencia de una posible invasión directa del virus, lo cual nos plantea la búsqueda apresurada de un posible potencial neuroinvasivo, aún poco esclarecido. En los casos crónicos la mayoría de síntomas neuropsiquiátricos se podrían explicar debido a que la inflamación acompaña a la mayoría de enfermedades neurodegenerativas. Finalmente, futuras investigaciones deben incluir diseños longitudinales (clínicos, neurológicos, neuropsiquiátricos, psicopatológicos, cognitivos, neuroimagenológicos y estudios de líquido cefaloraquídeo) de los pacientes con infecciones agudas por el SARS-CoV-2 en búsqueda de establecer posibles causalidades.

\section{CONCLUSIONES}

La comprensión de la COVID-19 como una enfermedad que afecta al sistema nervioso es imprescindible para el manejo posterior de los sobrevivientes. A través de esta revisión se ha buscado explicar el posible potencial neuroinvasivo del SARSCoV-2. Al momento de la concepción de este artículo, hay escasas investigaciones que hallan hecho estudios anatomopatológicos de partículas de SARS-CoV-2 en el sistema nervioso de los pacientes, así como estudios sobre su potencial neurotrópico junto con la respuesta inmunológica e inflamatoria del huésped, por ende, se deben realizar más estudios para dilucidar correctamente el tema y su relación con la evolución de los pacientes que presenten alteraciones neurológicas o psiquiátricas secundarias a la infección de SARSCoV-2.

Contribuciones de autorías: RA participó en la concepción del artículo; RA y JHV realizaron el diseño, redacción y la revisión crítica del artículo y aprobación de la versión final.

Fuentes de financiamiento: Autofinanciado. 
Conflicto de intereses: JHV realiza consultorías para la compañía Tecnofarma S.A. y Janssen Pharmaceutic.

\section{Correspondencia:}

Jeff David Huarcaya Victoria

Universidad de San Martín de Porres - Instituto de Investigación

Av. Alameda del Corregidor, 1531, La Molina, Lima Teléfono: 511-3653640

Correo electrónico: jhuarcayav@usmp.pe

\section{REFERENCIAS BIBLIOGRÁFICAS}

1. World Health Organization. WHO Coronavirus Disease (COVID-19). Ginebra: World Health Organization; 2020. (Citado el 9 de marzo del 2021) Disponible en: https://covid19. who.int/?gclid=CjwK CAiAqJn9BRB0EiwAJ1Sztem0DuIa_N2Ks2Ypc EOGgyyvJIIuRLQTimQ0xkrfjR-OY0sfiF 911BoC1sQQAvD_BwE

2. Huang $\mathrm{C}$, Wang $\mathrm{Y}$, Li X, Ren L, Zhao J, Hu $\mathrm{Y}$, et al. Clinical features of patients infected with 2019 novel coronavirus in Wuhan, China. Lancet. 2020;395(10223):497-506.

3. Lu Y, Li X, Geng D, Mei N, Wu PY, Huang CC, et al. Cerebral Micro-Structural Changes in COVID-19 Patients - An MRI-based 3-month Follow-up Study. EClinicalMedicine. 2020;25:100484.

4. Zubair AS, McAlpine LS, Gardin T, Farhadian S, Kuruvilla DE, Spudich S. Neuropathogenesis and Neurologic Manifestations of the Coronaviruses in the Age of Coronavirus Disease 2019: A Review. JAMA Neurol. 2020;77(8):1018-27.

5. Li K, Wohlford-Lenane C, Perlman S, Zhao J, Jewell AK, Reznikov LR, et al. Middle East Respiratory Syndrome Coronavirus Causes Multiple Organ Damage and Lethal Disease in Mice Transgenic for Human Dipeptidyl Peptidase 4. J Infect Dis. 2016; 213(5): 712-22.

6. Kim JE, Heo JH, Kim HO, Song SH, Park SS, Park $\mathrm{TH}$, et al. Neurological Complications during Treatment of Middle East Respiratory Syndrome. J Clin Neurol. 2017;13(3):227-33.

7. McCray PB, Pewe L, Wohlford-Lenane C, Hickey M, Manzel L, Shi L, et al. Lethal infection of K18hACE2 mice infected with severe acute respiratory syndrome coronavirus. J Virol. 2007;81(2):813-21.

8. Cheng Q, Yang Y, Gao J. Infectivity of human coronavirus in the brain. EBioMedicine. 2020;56:102799.

9. Tso EY, Tsang OT, Choi KW, Wong TY, So MK, Leung WS, et al. Persistence of physical symptoms in and abnormal laboratory findings for survivors of severe acute respiratory syndrome. Clin Infect Dis.
2004;38(9):1338.

10. Wu KK, Chan SK, Ma TM. Posttraumatic stress, anxiety, and depression in survivors of severe acute respiratory syndrome (SARS). J Trauma Stress. 2005; 18(1): 39-42.

11. Li Z, Liu T, Yang N, Han D, Mi X, Li Y, et al. Neurological manifestations of patients with COVID-19: potential routes of SARS-CoV-2 neuroinvasion from the periphery to the brain. Front Med. 2020;14(5):533-41.

12. Wiersinga WJ, Rhodes A, Cheng AC, Peacock SJ, Prescott HC. Pathophysiology, Transmission, Diagnosis, and Treatment of Coronavirus Disease 2019 (COVID-19): A Review. JAMA. 2020;324(8):782-93.

13. Hoffmann M, Kleine-Weber H, Schroeder S, Krüger N, Herrler T, Erichsen S, et al. SARS-CoV-2 Cell Entry Depends on ACE2 and TMPRSS2 and Is Blocked by a Clinically Proven Protease Inhibitor. Cell. 2020;181(2):271-80.

14. Mahase E. Covid-19: Russia approves vaccine without large scale testing or published results. BMJ. 2020;370:m3205. doi: 10.1136/bmj.m3205

15. Vaduganathan M, Vardeny O, Michel T, McMurray JJV, Pfeffer MA, Solomon SD. Renin-AngiotensinAldosterone System Inhibitors in Patients with Covid-19. N Engl J Med. 2020;382(17):1653-9.

16. Bestle D, Heindl MR, Limburg H, Van Lam van $\mathrm{T}$, Pilgram $\mathrm{O}$, Moulton $\mathrm{H}$, et al. TMPRSS2 and furin are both essential for proteolytic activation of SARS-CoV-2 in human airway cells. Life Sci Alliance. 2020;3(9):e202000786. doi: 10.26508/1sa.202000786

17. Chen R, Wang K, Yu J, Chen Z, Wen C, Xu Z. The spatial and cell-type distribution of SARS-CoV-2 receptor ACE2 in human and mouse brain. Front Neurol. 2021; 11: 573095. doi: 10.3389/ fneur.2020.573095

18. Mao L, Jin H, Wang M, Hu Y, Chen S, He Q, et al. Neurologic Manifestations of Hospitalized Patients With Coronavirus Disease 2019 in Wuhan, China. JAMA Neurol. 2020;77(6):683-90.

19. Lechien JR, Chiesa-Estomba CM, De Siati DR, Horoi M, Le Bon SD, Rodriguez A, et al. Olfactory and gustatory dysfunctions as a clinical presentation of mild-to-moderate forms of the coronavirus disease (COVID-19): a multicenter European study. Eur Arch Otorhinolaryngol. 2020;277(8):2251-61.

20. Rogers JP, Chesney E, Oliver D, Pollak TA, McGuire P, Fusar-Poli P, et al. Psychiatric and neuropsychiatric presentations associated with severe coronavirus infections: a systematic review and meta-analysis with comparison to the COVID-19 pandemic. Lancet Psychiatry. 2020;7(7):611-27.

21. Troyer EA, Kohn JN, Hong S. Are we facing a crashing wave of neuropsychiatric sequelae of COVID-19? Neuropsychiatric symptoms and potential 
immunologic mechanisms. Brain Behav Immun. 2020;87:34-9.

22. Liberati A, Altman DG, Tetzlaff J, Mulrow C, Gøtzsche PC, Ioannidis JP, et al. The PRISMA statement for reporting systematic reviews and metaanalyses of studies that evaluate health care interventions: explanation and elaboration. PLoS Med. 2009; 6(7): e1000100.

23. Singal CMS, Jaiswal P, Seth P. SARS-CoV-2, More than a Respiratory Virus: Its Potential Role in Neuropathogenesis. ACS Chem Neurosci. 2020;11(13):1887-99.

24. Saleki K, Banazadeh M, Saghazadeh A, Rezaei N. The involvement of the central nervous system in patients with COVID-19. Rev Neurosci. 2020;31(4):453-6.

25. Garg RK, Paliwal VK, Gupta A. Encephalopathy in patients with COVID-19: A review. J Med Virol. 2021;93(1):206-222. doi: 10.1002/jmv.26207

26. Ashraf O, Young M, Malik KJ, Cheema T. Systemic Complications of COVID-19. Crit Care Nurs Q. 2020;43(4):390-9.

27. Berth SH, Leopold PL, Morfini GN. Virus-induced neuronal dysfunction and degeneration. Front Biosci (Landmark Ed). 2009;14:5239-59.

28. Baig AM, Sanders EC. Potential neuroinvasive pathways of SARS-CoV-2: Deciphering the spectrum of neurological deficit seen in coronavirus disease-2019 (COVID-19). J Med Virol. 2020; 92(10):1845-1857. doi: 10.1002/jmv.26105

29. Paniz-Mondolfi A, Bryce C, Grimes Z, Gordon RE, Reidy J, Lednicky J, et al. Central nervous system involvement by severe acute respiratory syndrome coronavirus-2 (SARS-CoV-2). J Med Virol. 2020;92(7):699-702.

30. Bender JE, León R, Mendieta MD, Rodríguez R, Velázquez LC. Infección por el SARS-CoV-2: de los mecanismos neuroinvasivos a las manifestaciones neurológicas. Anales de la Academia de Ciencias de Cuba. 2020. 10(2)0-0. (Citado el 9 de marzo del 2021) Disponible en: http://www.revistaccuba.cu/ index.php/revacc/article/view/855/862

31. Vaira LA, Salzano G, Deiana G, De Riu G. Anosmia and Ageusia: Common Findings in COVID-19 Patients. Laryngoscope. 2020;130(7):1787. doi: 10.1002/lary.28692

32. Bilinska K, Jakubowska P, Von Bartheld CS, Butowt R. Expression of the SARS-CoV-2 Entry Proteins, ACE2 and TMPRSS2, in Cells of the Olfactory Epithelium: Identification of Cell Types and Trends with Age. ACS Chem Neurosci. 2020; 11(11): 155562.

33. Pennisi M, Lanza G, Falzone L, Fisicaro F, Ferri R, Bella R. SARS-CoV-2 and the Nervous System: From Clinical Features to Molecular Mechanisms. Int J Mol Sci. 2020; 21(15):0-0.
34. Li YC, Bai WZ, Hashikawa T. The neuroinvasive potential of SARS-CoV2 may play a role in the respiratory failure of COVID-19 patients. J Med Virol. 2020; 92(6): 552-5.

35 . Turtle L. Respiratory failure alone does not suggest central nervous system invasion by SARS-CoV-2. J Med Virol. 2020;92(7):705-6.

36. de Freitas Ferreira ACA, Romão TT, Y SIM, Pupe C, Nascimento OJ. COVID-19 and herpes zoster coinfection presenting with trigeminal neuropathy. Eur J Neurol. 2020;27(9):1748-1750. doi: 10.1111/ ene. 14361

37. Spiegel M, Schneider K, Weber F, Weidmann M, Hufert FT. Interaction of severe acute respiratory syndrome-associated coronavirus with dendritic cells. J Gen Virol. 2006;87(Pt 7):1953-60.

38. Azkur AK, Akdis M, Azkur D, Sokolowska M, van de Veen $\mathrm{W}$, Brüggen $\mathrm{MC}$, et al. Immune response to SARS-CoV-2 andmechanisms ofimmunopathological changes in COVID-19. Allergy. 2020;75(7):1564-81.

39. Zhao JM, Zhou GD, Sun YL, Wang SS, Yang JF, Meng EH, et al. Clinical pathology and pathogenesis of severe acute respiratory syndrome. Zhonghua Shi Yan He Lin Chuang Bing Du Xue Za Zhi. 2003;17(3):217-21.

40. Baig AM, Khaleeq A, Ali U, Syeda H. Evidence of the COVID-19 Virus Targeting the CNS: Tissue Distribution, Host-Virus Interaction, and Proposed Neurotropic Mechanisms. ACS Chem Neurosci. 2020;11(7):995-8.

41. Dropulić B, Masters CL. Entry of neurotropic arboviruses into the central nervous system: an in vitro study using mouse brain endothelium. J Infect Dis. 1990;161(4):685-91.

42. Kim WK, Corey S, Alvarez X, Williams K. Monocyte/ macrophage traffic in HIV and SIV encephalitis. J Leukoc Biol. 2003;74(5):650-6.

43. Serrano-Castro PJ, Estivill-Torrús G, CabezudoGarcía P, Reyes-Bueno JA, Ciano Petersen N, AguilarCastillo MJ, et al. Impact of SARS-CoV-2 infection on neurodegenerative and neuropsychiatric diseases: a delayed pandemic? Neurologia 2020;35(4):245-51.

44. Tian Y, Rong L, Nian W, He Y. Review article: gastrointestinal features in COVID-19 and the possibility of faecal transmission. Aliment Pharmacol Ther. 2020;51(9):843-51.

45. Jin X, Lian JS, Hu JH, Gao J, Zheng L, Zhang YM, et al. Epidemiological, clinical and virological characteristics of 74 cases of coronavirus-infected disease 2019 (COVID-19) with gastrointestinal symptoms. Gut. 2020;69(6):1002-9.

46. Llaro-Sánchez MK, Gamarra-Villegas BE, CamposCorrea KE. Características clínico-epidemiológicas y análisis de sobrevida en fallecidos por COVID-19 atendidos en establecimientos de la Red SabogalCallao 2020. Horiz Med. 2020;20(2): e1229. DOI: 
Alarco R, Huarcaya-Victoria J.

10.24265/horizmed

47. Cortés ME. Digestive symptoms in coronavirus disease 2019 (COVID-19). Rev Gastroenterol Peru. 2020;40(1):100-101.

48. Mönkemüller K, Fry L, Rickes S. COVID-19, coronavirus, SARS-CoV-2 and the small bowel. Rev Esp Enferm Dig. 2020;112(5):383-8.
49. Bostanciklıoğlu M. Temporal Correlation Between Neurological and Gastrointestinal Symptoms of SARS-CoV-2. Inflamm Bowel Dis. 2020;26(8): e89-e91.

Recibido: 05/12/2020 Aceptado: 16/03/2021 